DOI: $10.3724 /$ SP.J.1218.2011.00237

\title{
仿蟹机器人交错等相位波形步态研究
}

\author{
王 刚, 张立勋, 王立权 \\ (哈尔滨工程大学机电工程学院, 黑龙江 哈尔滨 150001)
}

\begin{abstract}
摘 要: 在观察生物螃蟹的基础上, 研制了仿蟹机器人实验样机. 利用事件序列法对生物螃蟹的行走过程进行 了分析, 提出了仿蟹机器人交错等相位波形步态. 交错等相位波形步态以 $2 n$ 足波形步态为基础, 将 8 个步行足交错 分为两组, 通过调节组内先后摆动的两步行足间的相位因子, 使同组的各步行足相位平均分配在一个步态周期内, 以减小机体能量损耗. 仿蟹机器人样机行走步态实验结果表明, 交错等相位波形步态符合生物螃蟹的运动特点, 与 双四足步态相比, 机器人具有良好的行走稳定性和较小的驱动功率波动幅度, 步行状态平稳.
\end{abstract}

关键词: 仿蟹形机器人; 交错等相位波形步态; 事件序列法; 步态规划

中图分类号: TP242

文献标识码: A

文章编号: 1002-0446(2011)-02-0237-07

\section{On Alternating Equal-Phase Wave Gait of Crab-like Robot}

\author{
WANG Gang, ZHANG Lixun, WANG Liquan \\ (College of Mechanical and Electrical Engineering, Harbin Engineering University, Harbin 150001, China)
}

\begin{abstract}
Based on bio-observation and analysis on natural crabs, a crab-like robot's prototype is developed. An alternating equal-phase wave gait for crab-like robot is presented by analyzing crabs' motion with event sequence method. Based on $2 n$-legged wave gait, the 8 legs are alternately divided into 2 groups, and the legs' phases in the same group are distributed uniformly within a circle by adjusting the phase factor of the successive legs within a tetrapod group in order to reduce energy consumption of the robot. The gait test results of the crab-like prototype show that the alternating equal-phase wave gait accords with motion characteristics of crabs, and the robot can walk smoothly and receive more favorable gait stability and smaller driving power fluctuation than the double tetrapod one.
\end{abstract}

Keywords: crab-like robot; alternating equal-phase wave gait; event sequence method; gait planning

\section{1 引言 (Introduction)}

在自然界中, 无论是哺乳类动物、爬行类动物 还是节肢类动物, 它们的行为方式多表现为一种多 步行足的协调运动, 这种协调运动通常称为步态. 步态是生物为了配合其机体实现从当前位置到目标 位置移动所完成的各步行足间抬腿和落腿的顺序关 系 ${ }^{[1]}$. 从步态的定义不难发现, 步态种类随步行足 数量的增加而增多, 与之相对应的机器人步态规划 问题也就变得更加复杂. 文 [2] 给出步行足数 $N>5$ 的足式机器人在数学上可实现的步态种类的计算通 式. 尽管理论上存在的步态形式很多, 但并非每种 步态都可以使机体有效地运动, 因此需要进行必要 的步态规划.

1961 年, Tomovic 和 Karplus 首次采用有限状态 理论分析了足式机器人的运动过程, 提出了摆动相
和支撑相概念 ${ }^{[3]}$. McGhee 比较系统地给出了关于 步态的一系列描述方法和步态分析的数学定义, 证 明了具有最大稳定裕度的四足机器人最优步态的存 在性 ${ }^{[4]}$. Song 总结了多足步行机器人的步态方法, 将步态划分为规则和非规则两大类，针对四足和六 足机器人, 提出了波形步态、等相位步态等 6 种规 则步态, 并分析了以自由步态为主的 4 种非规则步 态方法 ${ }^{[5]}$.

迄今为止, 国内外关于多足机器人步态的研究

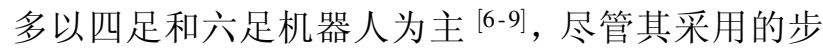
态一般都可以扩展到 $2 n(n>3)$ 足机器人, 但并未 针对性地给出具体的步态构造形式. 仅对八足仿蟹 机器人来说, 目前除双四足步态 ${ }^{[10-11]}$ 外, 并未提出 其它仿生步态形式，但双四足步态仅为生物蜳蟹行 走过程的一个特例, 不能代表生物螃蟹的运动规律, 
因此, 有必要对生物螃蟹的步态方法进行总结, 归 纳出真正意义上的仿生步态. 本文在深入分析生物 螃蟹运动步态的基础上, 提出了仿蟹机器人交错等 相位波形步态, 并对该步态方法进行了实验研究.

\section{2 仿蟹机器人样机（Prototype of crablike robot)}

在观察和研究生物螃蟹的基础上, 研制仿蟹机 器人样机, 如图 1 所示. 机器人样机由躯体架、6 条 运动步行足、 2 条复合作业步行足以及控制系统 4 部分构成, 初始状态下, 躯体平台距地面 $135 \mathrm{~mm}$, 呈近似 M 形.

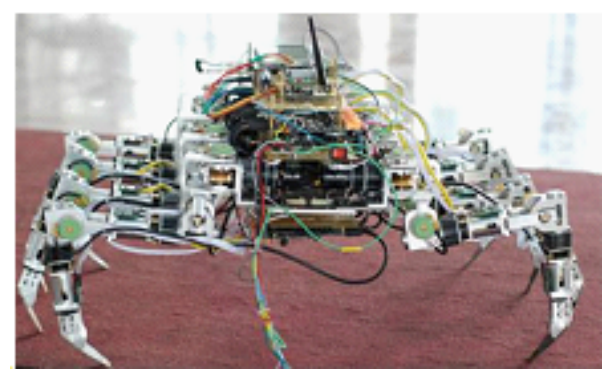

图 1 仿蟹机器人样机

Fig.1 Prototype of crablike robot

如图 2 所示, 步行足采用模块化结构, 由基节、 股节、胫节、指节、应力传感器及连接架等部分组 成, 其中胫节和指节彼此不存在相对运动, 简化为 一体, 即: 将每条步行足分解为 3 个模块化的传动 关节. 每个模块关节都由一个带减速器的电机和蜗 轮蜗杆传动机构构成, 为满足模块化关节传动系统 间隙小、刚度大、输出扭矩高、具有自锁性等要求, 选择传动效率高的圆弧圆柱蜗杆 (ZC) 传动方式.

依照步行足灵活度最优化比例 $l_{1}: l_{2}: l_{3}=0.05$ : $0.45: 0.50^{[12]}$, 步行足各部分尺寸及关节运动范围为 基节长度 $18 \mathrm{~mm}$, 髋关节转角范围 $-45^{\circ} \sim 45^{\circ}$; 股 节长度 $160 \mathrm{~mm}$, 膝关节摆动角范围 $-90^{\circ} \sim 90^{\circ}$; 胫 节长度 $175 \mathrm{~mm}$, 胫关节转角范围 $-135^{\circ} \sim 135^{\circ}$.

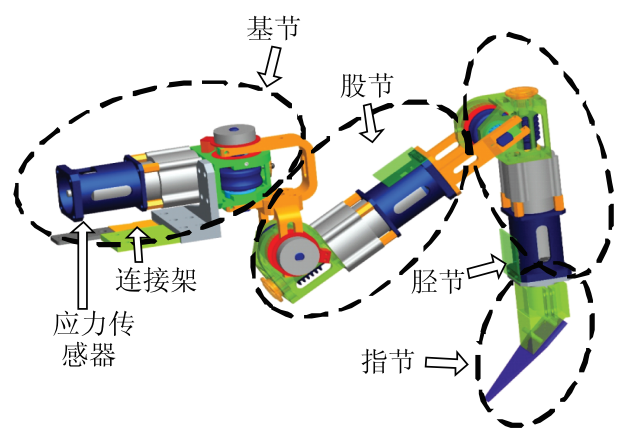

图 2 仿蟹机器人步行足结构

Fig.2 Walking leg structure of crablike robot
采用弹簧钢材料设计的扁形弹性足尖 (指节) 可 以使机器人在行走过程中更好地适应复杂地貌, 为 迈步提供额外作用力. 该足尖可以缓解机器人刚性 着地的能量损失和冲击, 并为下一步运动储存能量. 在足尖内表面粘贴应变片, 用于采集机器人行走时 的受力情况. 两条复合作业步行足在普通步行足基 础上安装作业机械手, 作业手采用双支点回转型手 爪设计思想, 利用一个蜗杆带动两个旋向相同的蜗 轮来实现, 由微型伺服电机驱动.

\section{3 步态规划 (Gait planning)}

步态的选择取决于机器人运动表面的平坦程 度、运动稳定性、灵活性以及能耗要求等多方面因 素. 在以往的研究中, 足式步行机器人通常选择波 形步态作为其基本运动模式, 以获得最佳的稳定边 界 ${ }^{[12]}$. 波形步态是一种对称规则步态, 也是许多动 物所采用的步态形式, 其处于躯体对称轴两侧的步 行足相位相差半个步态周期, 同侧步行足按其在躯 体上的排列顺序由后至前依次抬起和落下 ${ }^{[13-14]}$.

\section{1 步态描述}

\subsection{1 步态描述的基本参数}

(1) 摆动相 (transfer phase): 步行足处于摆动状 态的持续时间 $T_{\text {transfer }}$;

(2) 支撑相 (support phase): 步行足的着地持续 时间 $T_{\text {support }}$;

(3) 步态周期 (cycle time): 全部步行足完成一 次完整的步态循环的时间 $T$;

(4) 占空比 $\beta_{i}$ : 在一个步态周期内, 第 $i$ 条步行 足处于支撑相状态的时间与该步行足运动周期之 比:

$$
\beta_{i}=\frac{\text { support time of leg } i}{\text { cycle time of leg } i}=\frac{T_{\text {support }}^{i}}{T^{i}}
$$

(5) 步行足占地系数 $\beta$ : 单腿在地面支撑时间和 全部步行足完成一次步态循环时间之比:

$$
\beta=\frac{\text { legs' support time }}{\text { cycle time }}=\frac{T_{\text {support }}}{T}
$$

(6) 相对相位 $\varphi_{i}$ : 在一个步态周期中, 第 $i$ 条步 行足着地时刻 $t_{i}$ 滞后于第 1 条步行足着地时刻 $t_{1}$ 的 时间占整个步态周期的比例:

$$
\varphi_{i}=\frac{t_{i}-t_{1}}{T}
$$

\subsection{2 步态分析的事件序列法}

步态分析的描述方法包括: 时序图法、运动图 像法、连续步态模式法及足点图示法等 ${ }^{[5]}$. 这些方 法广泛应用于步态的分析和研究中, 特别是时序图 
法可以直观地描述各步行足的时间关系. 然而, 这 些方法都不能有效地描述生物自由运动时的步态 规律, 特别是当步行足数大于 6 时, 如果各步行足 的运动不具有明显的周期性, 则难以归纳出步行足 的运动规律. 为此, 本文提出一种新的步态描述方 法一一事件序列法.

所谓事件序列法, 就是将 $2 n$ 足生物的各步行足 抬腿事件与落腿事件以及相继抬腿的步行足间的时 间关系反映在一串事件序列上，以此建立事件序列 模型, 如式 (4).

$$
\begin{aligned}
& \cdots \rightarrow{ }^{b} K_{i}^{p_{i}} \underset{\ldots{ }^{b} K_{m}^{p_{m}} \cdots}{\stackrel{p_{j}-p_{i}}{b}} K_{j}^{p_{j}} \rightarrow \cdots \\
& i, j, m=1, \cdots, n \quad b=0,1
\end{aligned}
$$

其中, 当 $b=0$ 时, ${ }^{b} K=R$ 表示右侧步行足，当 $b=1$ 时, ${ }^{b} K=L$ 表示左侧步行足. 则上式表示左侧（或右 侧）第 $i$ 条步行足在 $p_{i}$ 时刻抬起，经 $p_{j}-p_{i}$ 个时间 单元后, 左侧（或右侧）第 $j$ 条步行足在 $p_{j}$ 时刻抬 起, 在此期间, 左侧 (或右侧) 第 $m$ 条步行足在 $p_{m}$
时刻落地, 依此类推获得整组步态的事件序列.

事件序列法可以有效地反映同侧、异侧以及整 个机体步行足间抬腿与落腿的时间顺序关系, 可以 完整地描述步行足间的运动规律, 直观地求出各步 行足的相对相位 $\varphi_{i}$ 以及占空比 $\beta_{i}$.

\section{2 生物螃蟹步态分析}

螃蟹作为甲壳纲十足目中较具代表性的动物之 一, 其运动以爬行为主, 大多数螃蟹采用近似横向 行走方式而不是直向行走方式, 并且它的步态运行 平稳且震动小 ${ }^{[15-16]}$. 从进化论角度来看, 螃蟹作为 一种经历过亿万年自然选择的生物, 它的步态形式 是逼近最佳的结果. 因此, 对于仿蟹形机器人步态 的研究首先从螃蟹的生物观察开始.

为了准确地记录螃蟹在平坦表面的行走模式, 采用摄像机对螃蟹的步行过程进行记录 (录像频率 30 帧/秒), 并在计算机中对录像进行采样 (采样频 率 5 帧(次), 标记每条步行足抬腿和落腿的时间, 图 3 所示为生物螃蟹步态时序图.

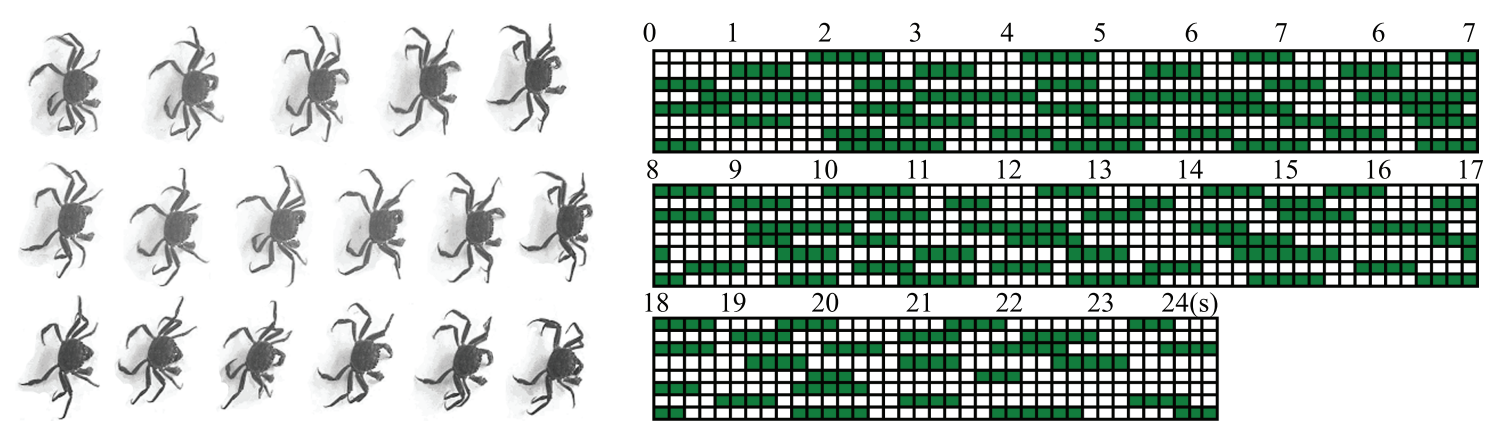

图 3 生物螃蟹步态及其时序图

Fig.3 Crab gaits and its sequence diagram

图 3 记录了螃蟹在平坦运动表面以较为自由的 步态行进时的运动时序. 在步态时序图中, 着色段 表示步行足处于摆动相, 空白段表示步行足处于支 撑相, 时序图的横坐标代表时间 $(\mathrm{s})$, 纵坐标代表 步序 (由上至下, 依次为左侧: $L_{1} 、 L_{2} 、 L_{3} 、 L_{4}$, 右 侧: $\left.R_{1} 、 R_{2} 、 R_{3} 、 R_{4}\right)$, 其步行的总时长为 $24 \mathrm{~s}$, 每个 小单元为 5 帧 $(1 / 6 \mathrm{~s})$.

通过对螃蟹的运动观察, 可以得出如下结论:

(1) 同侧步行足运动规律

螃蟹在运动过程中呈现一种相对自由的步态形 式, 但对于同侧的 4 条步行足来说, 它们总会依照 某一特定步态规则依次抬起和落下. 根据事件序列 法, 将生物螃蟹的运动时序图转换为事件序列, 图 4 所示为螃蟹左侧和右侧步行足的步态事件序列.

根据生物螃蟹的运动规律, 将步行足的运动时 序分解为起始期、稳定期和过渡期 3 个部分, 则右
侧步行足的运动时序分别为:

$$
\begin{aligned}
& R\{2341-2314-2314-2314-2314-2314-2314- \\
& \quad 2312-4341-2342-1314-34\}
\end{aligned}
$$

右侧 4 条步行足的起始时序为 $R-2341$, 假设步 行足运动时序具有周期性, 令 $R_{4}$ 为起始足, 则起始 时序可记为 $R$-4123. 随后进入稳定期, 此时运动时 序变为 $R-4231$, 并稳定持续 6 个周期. 经过前 7 个 运动周期后, 步行足的运动形式发生突变, 进入过 渡期, 步行足运动时序失去原规律, 不难理解, 在过 渡期, 螃蟹的某些步行足由于外部干扰导致其失去 原有步态平衡. 因此, 对于生物螃蟹右侧的步行足 来说, 其步态规则以 $R-4231$ 为主. 同理建立左侧步 行足的事件序列, 经分析, 其具有相类似的规律.

(2) 对侧步行足运动规律

对侧同序步行足 (图 3 中用相同颜色表示摆动 
相的两个步行足）一般不满足轴对称步行机纵向运 动规则, 即对称步态的轴对称步行足对相位差半个 周期的假设, 因此螃蟹运动时采用的并不是严格的 波形步态.

$$
\begin{aligned}
& R_{2}^{5} \frac{6}{R_{2}^{9}} R_{3} \frac{1}{R_{4}^{12}} \frac{1}{1} R_{1}^{13} \frac{3}{R_{3}^{15}} R_{2}^{16} \frac{6}{R_{3}^{19}} R_{3}^{22} \frac{3}{R_{1}^{25}} \frac{1}{R_{3}^{26}} \\
& R_{4}^{26} \frac{2}{R_{2}^{9}} R_{3}^{28} \frac{6}{R_{2}^{29} ; R_{2}^{33}} R_{3}^{34} \frac{3}{37} R_{1}^{37} \frac{1}{R_{3}^{38}} R_{4}^{38} \frac{3}{2} R_{2}^{41} \frac{3}{R_{1}^{42}} R_{3}^{44} \frac{5}{R_{2}^{45} ; R_{2}^{48}} \\
& R_{1}^{49} \frac{1}{-50} R_{4}^{50} \frac{0}{-50} \frac{6}{R_{2}^{55} ; R_{4}^{56}} R_{3}^{56} \frac{1}{R_{3}^{60}} R_{1}^{60} \frac{2}{-62} R_{4}^{62} \frac{0}{R_{1}^{42}} R_{2}^{62} \frac{5}{R_{4}^{66} ; R_{2}^{66}} \\
& R_{3}^{67} \frac{0}{-67} \frac{3}{R_{1}^{70}} R_{4}^{70} \frac{1}{R_{3}^{71}} R_{2}^{71} \frac{5}{R_{4}^{75} ; R_{2}^{75}} R_{3}^{76} \frac{1}{-} R_{1}^{77} \frac{4}{R_{3}^{80}} R_{4}^{81} \frac{0}{-} \\
& R_{2}^{81} \frac{5}{R_{1}^{82} ; R_{2}^{85}} R_{3}^{86} \frac{5}{R_{3}^{90}} R_{1}^{91} \frac{1}{-} R_{2}^{92} \frac{1}{-} R_{4}^{93} \frac{7}{R_{1}^{96} ; R_{4}^{98} ; R_{2}^{99}} R_{3}^{100} \frac{4}{R_{3}^{104}} \\
& R_{4}^{104} \frac{1}{-105} R_{1}^{2}-R_{2}^{107} \frac{5}{R_{1}^{108} ; R_{4}^{110} ; R_{2}^{111}} R_{3}^{112} \frac{1}{R_{3}^{116}} R_{4}^{117} \underline{0} R_{2}^{117} \frac{1}{-13} \\
& R_{1}^{118} \frac{6}{R_{1}^{121} ; R_{4}^{122} ; R_{2}^{122}} R_{3}^{124} \frac{5}{R_{3}^{128}} R_{1}^{129} \frac{1}{-130} \frac{9}{R_{1}^{132} ; R_{4}^{136}} R_{3}^{139} \frac{3}{{ }^{142}} R_{4}
\end{aligned}
$$

图 4 螃蟹右侧步行足事件序列

Fig.4 Event sequence diagram of crab's right legs

(3) 螃蟹在自由爬行时, 由于几对步行足长度不 同, 其行进方向通常朝向侧前方, 而不是绝对的纵 向或横向, 但螃蟹的行走可认为是一种近似的横行, 螃蟹近似横向行走时, 其重心较低, 稳定性好.

(4) 通常情况下, 螃蟹在行进过程中一般至少有 4 条步行足同时处于支撑相状态, 只有在疾走时, 允 许某个瞬时只有 3 足处于支撑相, 但仍满足 8 足步 态静态稳定的必要条件 $\beta \geqslant 3 / 8$, 而同侧步行足至 少有 1 个步行足处于支撑相. 当螃蟹的行进速度变 化时, 处于摆动相的各步行足的摆动时间变化不明 显, 发生变化的主要是各步行足的相对相位.

(5) 生物螃蟹的运动周期的平均值为 $2 \mathrm{~s}$, 其中, 摆动相所占比例的平均值为 $38.75 \%$, 支撑相所占比 例的平均值为 $61.25 \%$.

\section{3 交错等相位波形步态}

经分析, 生物螃蟹在平坦表面的运动步态可以 近似归纳为交错等相位波形步态, 如图 5 所示. 将 8 条步行足交错分组, 分成 $R_{4}-L_{3}-R_{2}-L_{1}$ 和 $L_{4}-R_{3}-L_{2}-R_{1}$, 同组的 4 条步行足由最后面的步行足起依次横向摆 动, 且先后摆动的两步行足相位相差 $\Delta \times \pi$, 其中 $\Delta$ 称为相位因子, 取值为 $0<\Delta<1$. 当 $\Delta \leqslant 1 / 3$ 时, 身区 体同侧的步行足摆动顺序为 $R-4231$ ( $L-4231)$; 随着 相位因子增大, 当 $\Delta>1 / 3$ 时, 躯体一侧的步行足 摆动顺序变为 4-1-2-3, 且当 $\Delta=0.5$ 时, 步态为双 四足步态. 如果 $\Delta$ 持续增大, 当 $\Delta>1$ 时, 步态形式 失去交错性, 变为波形步态.

尽管生物螃蟹的两组交错 4 足间的相位差是变
化的, 但在机器人步态的研究中, 为了获得最优的 步态稳定边界, 将相位差固定为半个周期 $T / 2$, 则 同侧相邻两步行足间的相位差为 $T / 2+\Delta \times \pi, \overrightarrow{R_{4} L_{3}}$ 等于 $\overrightarrow{L_{4} R_{4}}$ 和 $\overrightarrow{L_{4} L_{3}}$ 的向量差.

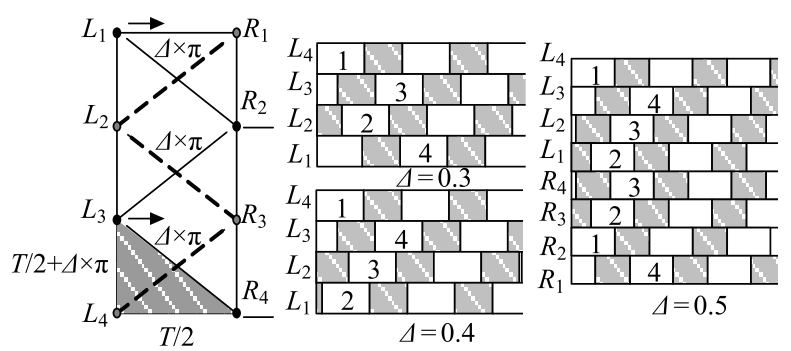

图 5 交错等相位波形步态实现方法

Fig.5 Realization method of the alternating equal-phase wave gait

为了使步态的能量损耗、能量波动小, 步行足 驱动力分布平均, 将同组的各步行足相位平均分配 在一个步态周期内, 相应地另一组步行足也平均分 配在一个步态周期内, 令两组间的相位相差 $T / 2$, 此 时同组的 4 个步行足构成等相位步态. 根据等相位 步态定义, $2 n$ 足步行机器人的相对相位 $\varphi_{i}$ 为

$$
\varphi_{2 m+1}=1-m / 2 n, \quad m=1,2 \cdots, n-1
$$

其中, $2 m+1$ 表示四足组中左侧步行足的序数, $2 n$ 为总步行足数. 对八足仿蟹机器人来说 $n=4$, 求得 $\varphi_{3}=1-1 / 8, \varphi_{5}=1-2 / 8, \varphi_{7}=1-3 / 8$, 即: 同组 同侧相邻步行足的相位差为 $\Delta \phi=2 \pi / 4$. 如图 5 中 $L_{2}$ 和 $L_{4}$ 的相位差为 $\Delta \phi=2 \Delta \times \pi$. 由此, 可求得等 相位步态时的相位因子 $\Delta=0.25$.

相位因子 $\Delta$ 给出了各步行足间的相位关系, 但 步行足占空比 $\beta_{i}$ 则给出了步行足支撑相和摆动相的 关系. 对于规则步态, 其所有步行足占空比相等且 等于整个步态的占地系数 $\beta$, 即 $\beta_{i}=\beta_{j}=\beta$. 为了 计算 $\beta_{i}$, 建立如下参数方程:

$$
\begin{aligned}
& T_{\text {transfer }}^{i}\left(T^{i}\right)=m_{\mathrm{T}} \times T^{i}+b_{\mathrm{T}} \\
& T_{\text {support }}^{i}\left(T^{i}\right)=m_{\mathrm{S}} \times T^{i}+b_{\mathrm{S}} \\
& \begin{aligned}
P_{i} & =T_{\text {transfer }}^{i} / T_{\text {support }}^{i} \\
& =\left(m_{\mathrm{T}} \times T^{i}+b_{\mathrm{T}}\right) /\left(\left(1-m_{\mathrm{T}}\right) T^{i}-b_{\mathrm{T}}\right) \\
\beta_{i} & =1 /\left(P_{i}+1\right)
\end{aligned}
\end{aligned}
$$

其中, $T_{\text {transfer }}^{i}$ 和 $T_{\text {support }}^{i}$ 分别代表支撑相和摆动相, 且 $T^{i}=T_{\text {transfer }}^{i}+T_{\text {support }}^{i}, m_{\mathrm{T}}$ 和 $m_{\mathrm{S}}$ 作为支撑相和摆动相 在一个周期内的比例因子, 决定了最低运动速度, 有 $m_{\mathrm{T}}+m_{\mathrm{S}}=1 . b_{\mathrm{T}}$ 和 $b_{\mathrm{S}}$ 是支撑相和摆动相的调整 因子, 且 $b_{\mathrm{T}}+b_{\mathrm{S}}=0$, 若 $b_{\mathrm{T}}=b_{\mathrm{S}}=0$, 则支撑相与摆 动相的比保持恒定. 用 $P$ 表示摆动相和支撑相的比, 
显然 $P$ 越小, 运动稳定性越好, 但移动速度也随之 变小 ${ }^{[17]}$.

通过对生物螃蟹进行运动时序分析, 步态参数 可近似为 $m_{\mathrm{T}}=0.31, m_{\mathrm{S}}=0.69, b_{\mathrm{T}}=160 \mathrm{~ms}, b_{\mathrm{S}}=$ $-160 \mathrm{~ms}$, 代入方程 (6) 和 (7), 分别计算各步行足摆 动 (支撑) 时间与周期的关系 (如图 6(a) 所示) 及摆 动相和支撑相的比与周期的关系 (如图 6(b) 所示).

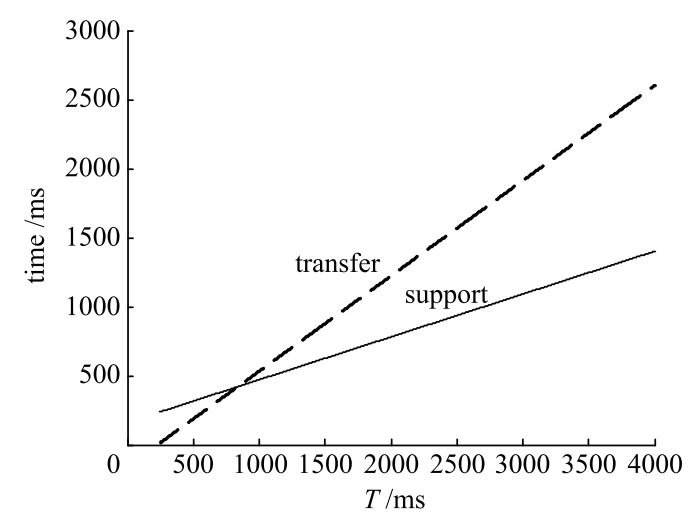

(a) 摆动时间和支撑时间与周期的关系

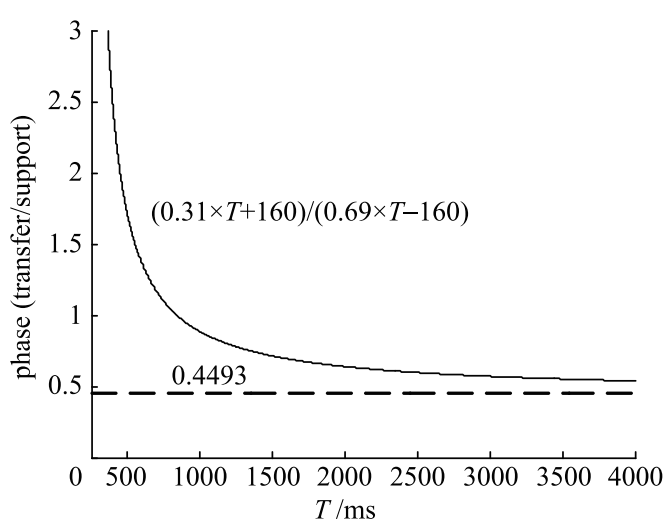

(b) 摆动相和支撑相的比与周期的关系

图 6 摆动相一支撑相与周期的关系曲线

Fig.6 Relationship curve between transfer-support phase and period

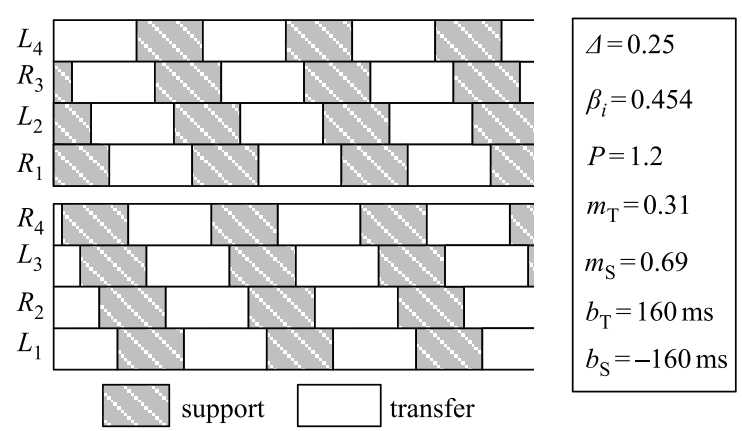

图 7 交错等相位波形步态时序图

Fig.7 Sequence diagram of alternating equal-phase wave gait

在保证运动稳定性的前提下, 为获得较大的移 动速度, 取 $P=1.2$, 代入方程 (8), 求得 $\beta_{i}=0.454$,
满足步态稳定性条件 $\beta \geqslant 3 / 8$.

图 7 所示为仿蟹机器人交错等相位波形步态时 序图及其参数选择.

\section{4 步态实验 (Gait testing)}

建立仿蟹机器人虚拟样机模型, 导入 ADAMS, 设定各部件的质量及惯量信息. 建立基于 MatlabSimulink 的控制系统模型, 通过位置一速度双闭环 系统控制电机参数, 对机器人交错等相位波形步态 的运动情况进行联合仿真, 设定机器人行走步长为 $160 \mathrm{~mm}$, 运动表面平坦.

机器人机体重心的变化情况以及机体步行速度 的稳定性是评价机器人运动稳定性的重要参数. 如 图 8 所示, 在运动过程, 采用交错等相位波形步态 运动的机器人质心起伏在 $1 \mathrm{~mm}$ 内, 而双四足步态 的质心起伏量为 $2.75 \mathrm{~mm}$. 相比传统的双四足步态, 交错等相位波形步态的质心浮动量降低了 $63 \%$.

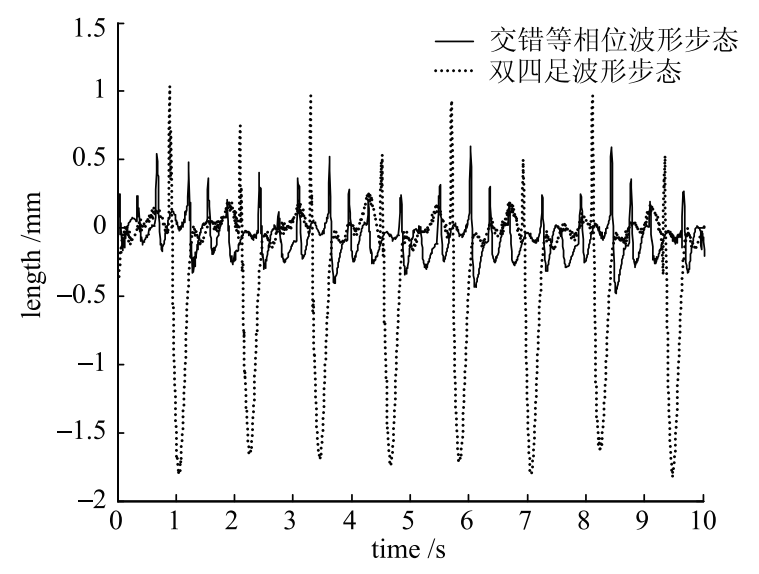

图 8 机器人质心波动曲线

Fig.8 Centroid fluctuation curve of the robot

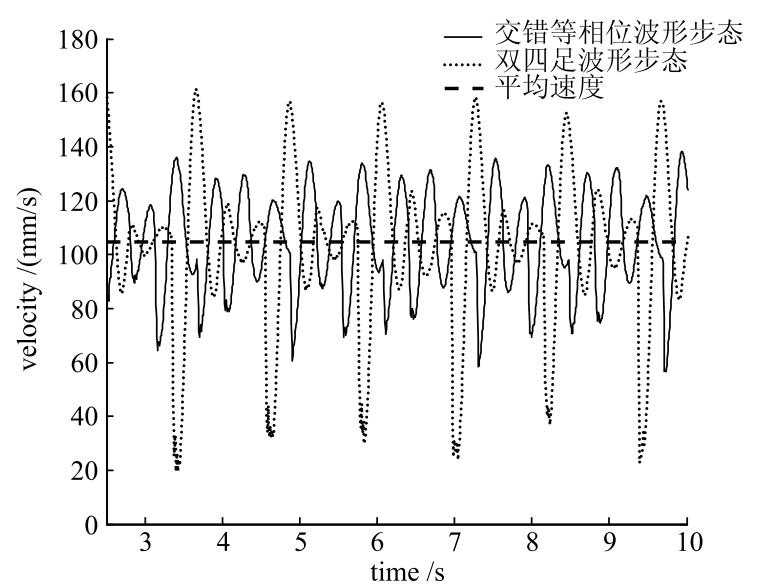

图 9 机体步行速度波动曲线

Fig.9 Velocity fluctuation curve of the body

采用交错等相位波形步态时，机体步行速度的 振荡幅度明显小于双四足步态, 如图 9 所示, 两种 
步态的平均速度为 $103 \mathrm{~mm} / \mathrm{s}$ (图中虚线部分), 交 错等相位波形步态的机体步行速度的振荡幅度在 $60 \mathrm{~mm} / \mathrm{s} \sim 140 \mathrm{~mm} / \mathrm{s}$, 小于双四足步态的 $20 \mathrm{~mm} / \mathrm{s} \sim$ $160 \mathrm{~mm} / \mathrm{s}$, 即机体速度振荡幅度下降了 $42.8 \%$, 明显 降低由于机体速度变化所引起的能量损耗. 因此, 对于仿蟹机器人, 在迈步周期和步幅确定的前提下, 交错等相位波形步态相对于双四足步态而言, 其行 走更加平稳.

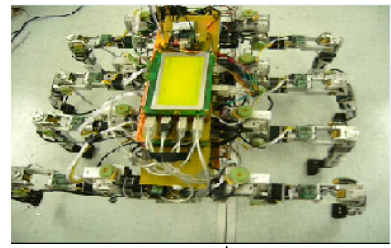

(1)

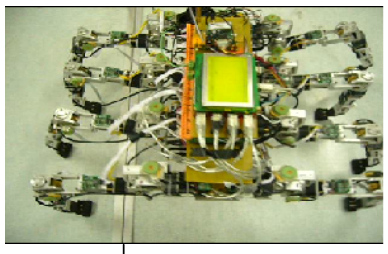

(3)

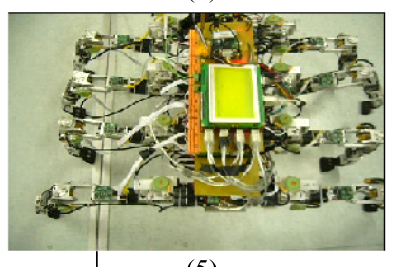

(5)

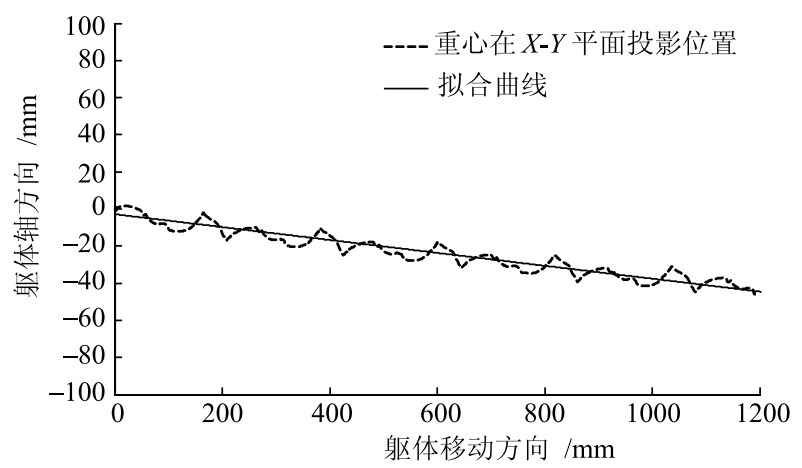

图 10 重心轨迹试验曲线

Fig.10 Trajectory curve of the gravity centre

图 10 为采用交错等相位波形步态行走的仿蟹 机器人运动测试图及重心轨迹试验曲线. 经测试, 交错等相位波形步态更接近生物螃蟹的运动特点, 机器人运动状态平稳, 其机体实际的移动方向为侧 前方, 偏斜角度为 $2^{\circ}$, 与生物观察结果接近.

测量交错等相位波形步态及双四足步态的实时 驱动电压和电流, 计算实时驱动功率, 从步态驱动 功率波动曲线来看双四足步态的能量波动较大, 且 峰值功率可达 $185 \mathrm{~W}$, 高于交错等相位波形步态的
$150 \mathrm{~W}$, 如图 11 所示. 由于仿蟹机器人一般采用电 池供电方式, 此时, 双四足步态消耗功率大, 要求电 池容量大, 这会导致电池体积和重量增加, 反而使 性能下降.

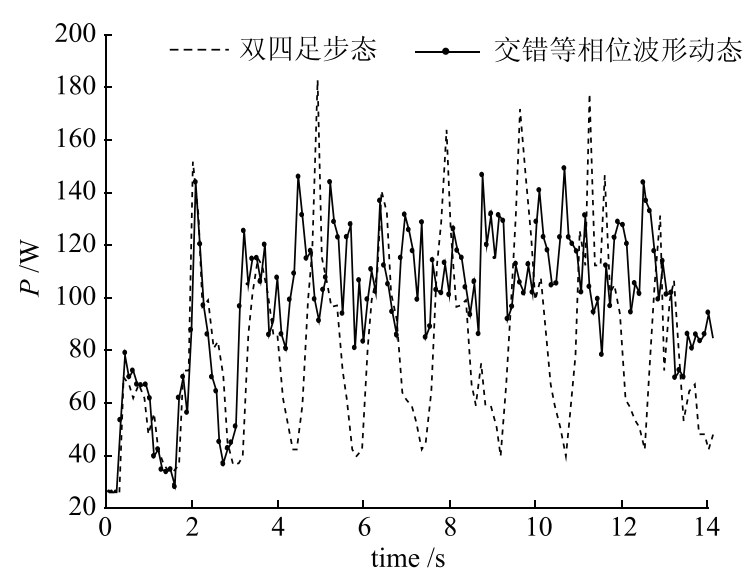

图 11 机器人驱动功率曲线

Fig.11 Driving power curve of the robot

\section{5 结论 (Conclusion)}

在观察和分析生物螃蟹行走步态的基础上，研 制了仿蟹形机器人实验样机, 进行了实验研究. 利 用事件序列法分析了生物螃蟹在平坦表面运动的 步态规律, 总结并提出了仿蟹机器人交错等相位波 形步态. 步态实验结果表明, 与传统的双四足步态 相比, 采用交错等相位波形步态的机器人其质心浮 动量降低了 $63 \%$, 机体步行速度的振荡幅度下降了 $42.8 \%$, 其实际步行方向为侧前方, 实时驱动功率波 动小, 该步态有效降低了由于机体运动速度交替变 化所带来的能量损耗, 步行状态平稳. 机器人样机 运动测试结果表明, 本文提出的交错等相位波形步 态符合生物螃蟹的运动特点, 对于仿蟹机器人的研 究具有实际意义.

\section{参考文献（References）}

[1] 陈学东, 孙翊, 贾文川. 多足步行机器人运动规划与控制 [M]. 武汉: 华中科技大学出版社, 2006.

Chen X D, Sun X, Jia W C. Motion planning and control of multilegged walking robots[M]. Wuhan: Huazhong University of Science and Technology Press, 2006.

[2] Bessonov A P, Umnov N W. Choice of geometric parameters of walking machines[C]//2nd CISM/IFTOMM International Symposium on the Theory and Practice of Robots and Manipulators. Amsterdam, Netherlands: Elsevier, 1977: 62-73.

[3] Tomovic R, Karplus W J. Land locomotion simulation and control[C]//3rd International Analogue Computation Meeting. Opatija, Yugoslavia: 1961: 385-390.

[4] McGhee R B. Some finite state aspects of legged locomotion[J]. Mathematical Biosciences, 1968, 2(1/2): 67-84.

[5] Song S-M, Waldron K J. Machines that walk: The adaptive suspension vehicle[M]. Cambridge, MA, USA: MIT Press, 1988. 
[6] Hirose S, Fukuda Y, Yoneda K, et al. Quadruped walking robots at Tokyo Institute of Technology: Design, analysis, and gait control methods[J]. IEEE Robotics and Automation Magazine, 2009, 16(2): 104-114.

[7] Howard A M, Parker L T. A hierarchical strategy for learning of robot walking strategies in natural terrain environments[C]// 2007 IEEE International Conference on Systems, Man and Cybernetics. Piscataway, NJ, USA: IEEE, 2008: 2336-2341.

[8] Nabulsi S, Sarria J F, Montes H, et al. High-resolution indirect feet-ground interaction measurement for hydraulic-legged robots[J]. IEEE Transactions on Instrumentation and Measurement, 2009, 58(10): 3396-3404.

[9] 郭丽峰, 陈恳, 赵旦谱, 等. 一种轮腿式变结构移动机器人 研究 [J]. 制造业自动化, 2009, 31(10): 1-6.

Guo L F, Chen K, Zhao D P, et al. Study on a wheel-legged hybrid mobile robot[J]. Manufacturing Automation, 2009, 31(10): $1-6$.

[10] 王立权, 刘德峰, 陈东良, 等. 两栖多足机器人水下步态分 析 [J]. 机器人, 2008, 30(4): 333-339.

Wang L Q, Liu D F, Chen D L, et al. Analysis on underwater gait of amphibious multi-legged robot[J]. Robot, 2008, 30(4): 333-339.

[11] 袁鹏. 仿生机器蟹步行机理分析及控制系统研究 [D]. 哈尔 滨: 哈尔滨工程大学, 2003.

Yuan P. Research on walking mechanism analysis and control system of a crab-liked biologically inspired robot[D]. Harbin: Harbin Engineering University, 2003.

[12] 韩宝玲, 王秋丽, 罗庆生. 六足仿生步行机器人足端工作 空间和灵活度研究 [J]. 机械设计与研究, 2006, 22(4): 1012,31 .
Han B L, Wang Q L, Luo Q S. Mechanical optimization and analyses of hexapod walking bio-robot[J]. Machine Design and Research, 2006, 22(4): 10-12,31.

[13] Wang P F, Sun L N. The stability analysis for quadruped bionic robot[C]//EEE/RSJ International Conference on Intelligent Robots and Systems. Piscataway, NJ, USA: IEEE, 2006: 5238-5242.

[14] Klaassen B, Linnemann R, Spenneberg A, et al. Biologically inspired robot design and modeling[C]//11th International Conference on Advanced Robotics. Coimbra, Portugal: University of Coimbra, 2003: 576-581.

[15] Estremera J, Cobano J A, Gonzalez de Santos P. Continuous free-crab gaits for hexapod robots on a natural terrain with forbidden zones: An application to humanitarian demining[J]. Robotics and Autonomous Systems, 2009, 58(5): 700-711.

[16] Vidal-Gadea A G, Rinehart M D, Belanger J H. Skeletal adaptations for forwards and sideways walking in three species of decapod crustaceans[J]. Arthropod Structure and Development, 2008, 37(2): 95-108.

[17] Klaassen B, Linnemann R, Spenneberg D. Biomimetic walking robot SCORPION: Control and modeling[J]. Robotics and Autonomous Systems, 2002, 41(2/3): 69-76.

\section{作者简介:}

王 刚 (1983-), 男, 博士生. 研究领域为: 智能仿生机器 人系统.

张立勋 (1962-), 男, 工学博士, 教授. 研究领域: 机器人 技术, 机电一体化技术.

王立权 (1957-), 男, 工学博士, 教授. 研究领域: 智能仿 生机器人系统, 水下智能机械, 机电一体化技术. 